\title{
Experimental studies of dark current in a superconducting rf photoinjector
}

\author{
R. Xiang, ${ }^{1, *}$ A. Arnold, ${ }^{1}$ T. Kamps, ${ }^{2}$ P. Lu, ${ }^{1,3}$ P. Michel, ${ }^{1}$ P. Murcek, ${ }^{1}$ \\ H. Vennekate, ${ }^{1,3}$ G. Staats, ${ }^{1}$ and J. Teichert ${ }^{1}$ \\ ${ }^{1}$ Helmholtz-Zentrum Dresden-Rossendorf, Bautzner Landstrasse 400, 01328 Dresden, Germany \\ ${ }^{2}$ Helmholtz-Zentrum Berlin, Albert-Einstein-Strasse 15, 12489 Berlin, Germany \\ ${ }^{3}$ Technische Universität Dresden, 01062 Dresden, Germany
}

(Received 6 December 2013; published 4 April 2014)

\begin{abstract}
A superconducting rf photoinjector (SRF gun) has been put in operation successfully at the radiation source ELBE. It produces a $13 \mathrm{MHz}$, low emittance, cw beam with beam current up to $400 \mu \mathrm{A}$ and energy of $3 \mathrm{MeV}$. During the gun operation, the field emission from the niobium cavity and the $\mathrm{Cs}_{2} \mathrm{Te}$ cathodes produces dark current. In this paper, we study the dark current of the SRF gun by using the existing diagnostics beam line. The dependency of the intensity and the energy spectrum on the cavity gradient and the cathode voltage are examined, and the emission sources are analyzed. Furthermore, the new project of installing a strip line kicker to reduce the dark current effect is presented.
\end{abstract}

DOI: 10.1103/PhysRevSTAB.17.043401

PACS numbers: 07.77.Ka, 79.70.+q, 85.60.Ha

\section{INTRODUCTION}

The injector plays a significant role in accelerator facilities for free-electron laser (FEL) light sources, energy recovery linacs (ERLs), electron colliders, and Thomson backscattering sources [1-4]. In recent years, superconducting rf photoinjectors (SRF gun) have drawn a lot of attention because of their characters: continuous-wave (cw) operation, flexible bunch structures, low emittance compared to the other high average current sources [5] and the potential application for polarized beam generation [6]. The ELBE SRF gun within the cooperation of HZDR, HZB, DESY and MBI has been successfully commissioned [7,8] and firstly operated for the ELBE IR-FEL [9]. It is operated at a peak field $E_{z \cdot \max }$ of up to $16.2 \mathrm{MV} / \mathrm{m}$ at cw mode and $21.6 \mathrm{MV} / \mathrm{m}$ at macropulse mode. With a $\mathrm{Cs}_{2} \mathrm{Te}$ photocathode [10] driven by a $13 \mathrm{MHz}$ UV laser, the ELBE SRF gun has produced photocurrent beams of up to $400 \mu \mathrm{A}$.

During the operation of an $\mathrm{rf}$ photoinjector with high gradient, not only the photoelectron beam but also dark current is produced. The dark current is a part of field emission from the cavity and photocathodes, which is accelerated through the gun together with the photocurrent beam. It belongs to unwanted beam, which can lead to beam loss, increase the risk of damage to accelerator components, and create additional background for primary or radiation beam users. To understand the dark current of normal conducting (NC) rf guns, a lot of measurements have been done at DESY, SLAC and FermiLab [11-14]. Some models

\footnotetext{
"r.xiang@hzdr.de

Published by the American Physical Society under the terms of the Creative Commons Attribution 3.0 License. Further distribution of this work must maintain attribution to the author $(s)$ and the published article's title, journal citation, and DOI.
}

and simulations were developed to provide the physical insight on field emission in the rf guns [14-17]. Ultrafast beam line components were designed to help detect and control the dark current [18].

For superconducting rf guns, the cavity gradient is not as high as in NC rf guns, but for the cw operation the dark current cannot be ignored [19]. In this paper we will present our experimental studies on the dark current at the ELBE SRF gun. The dark current as a function of the cavity gradient and the cathode voltage is monitored and the energy spectra of the dark current are examined. Based on the experimental data, we analyze the origin of the dark current in the gun according to the Fowler-Nordheim (F-N) theory. At last we discuss the option to decrease the dark current at the exit of the gun.

\section{CAVITY GEOMETRY AND FIELD DISTRIBUTION OF SRF GUN}

As the key part of the ELBE SRF gun, the $3+1 / 2$ cell niobium cavity, operated at $1.3 \mathrm{GHz}$, consists of three TESLA cells and one specially designed half cell. The ratio of the peak axis field to the efficient accelerating field $E_{z \cdot \max } / E_{\text {acc }}=2.70$ [7]. A normal conducting cathode plug is inside of an adopted cathode hole in the half cell, isolated with a vacuum gap. The diameter of the plug is $10 \mathrm{~mm}$ while the diameter of the cathode hole is $12 \mathrm{~mm}$. In order to suppress multipacting appearing in the gap, a cathode biasvoltage 3-6 kV is applied on the plug. The $\mathrm{Cs}_{2} \mathrm{Te}$ layer (diameter $8 \mathrm{~mm}$ ) deposited on the molybdenum plug is the standard photocathode for this gun. More details are described in [7].

In Fig. 1, the distribution of the electrical surface field $|E|$ on the cavity wall and the z component of electric field $E_{z}$ on axis are presented along the cavity's $\mathrm{z}$ axis [20]. The maximum axis field $E_{z \cdot \max }$ is set to $16.2 \mathrm{MV} / \mathrm{m}$. For a 


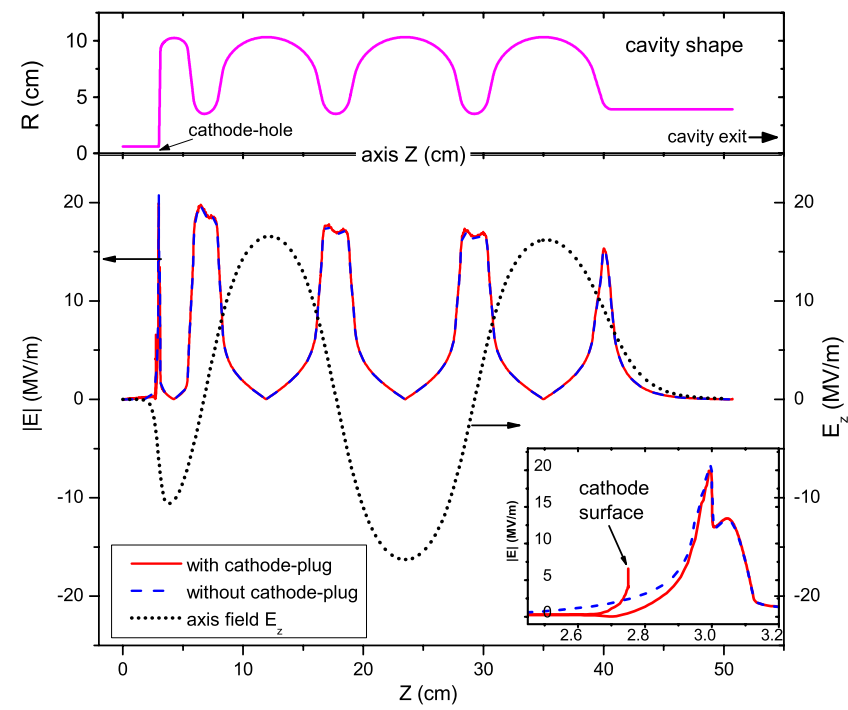

FIG. 1. Top: The cavity shape of the ELBE SRF gun. Bottom: The distribution of the electric surface field $|E|$ and the axis field $E_{z}$, simulated using SUPERFISH [21] with $E_{z \cdot \max }=16.2 \mathrm{MV} / \mathrm{m}$. The solid red line and the blue dashed line are respectively the case with cathode plug or without cathode plug, while the black dots show the acceleration field $E_{z}$ along the $z$ axis (without cathode). Inset: The distribution of the electric surface field in the zone close to the cathode $(z=2.6 \mathrm{~cm}$ to $3.2 \mathrm{~cm})$ [20].

better understanding, the cavity shape is shown in the upper part of the figure, and in the inset is the zoomed zone close to the cathode $(\mathrm{z}=2.6$ to $3.2 \mathrm{~cm})$. Obviously, the presence of a cathode does not change the field distribution in the TESLA cells and the peak field in the half cell. It is worthy to note that the maximum surface field in the half cell locates at the edge of the cathode hole, where the field amplitude reaches $20 \mathrm{MV} / \mathrm{m}$, about $120 \%$ of the peak field on axis $E_{z \text { max }}$. If a cathode is inside the cavity hole, the maximum field on the cathode's surface reaches $6.5 \mathrm{MV} / \mathrm{m}$.

From the surface field $|E|$ distribution, the potential field emission areas are located at the edge of the cathode hole, the cavity iris, and the back wall of the half cell. However, the field emission around the cathode hole composes the main part of the dark current, where the field emitted electrons can be efficiently accelerated forward by $E_{z}$. The field emitted electrons from the iris experience only a quite weak $E_{z}$, and may be trapped in the cavity. On the other hand, the trapped electrons can hit the cavity wall, leading to more helium consumption and causing the emission of secondary electrons; also they can back-bombard the cathode layer, degrading the cathode's quality.

\section{DARK CURRENT MEASUREMENT}

\section{A. Experimental setup}

The setup of our experiment consists of the SRF gun, various photocathodes, the cathode transport system, the

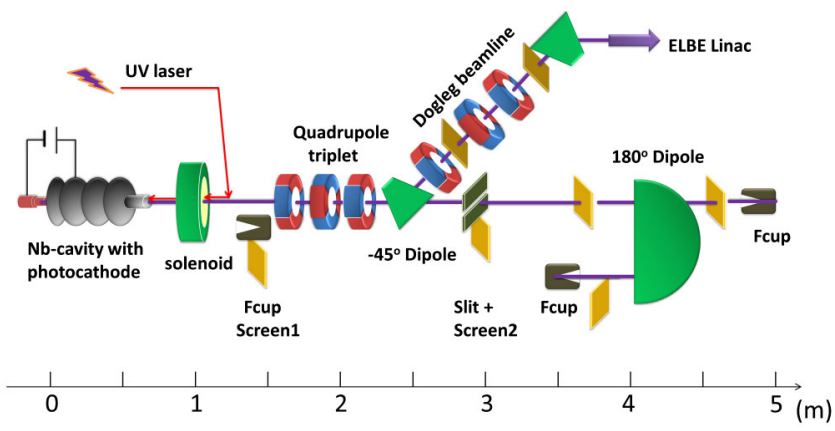

FIG. 2. The schematic view of the gun, the diagnostic beam line, and the dogleg beam line connecting the setup to the ELBE linac. The $-45^{\circ}$ dipole, $2.39 \mathrm{~m}$ downstream from the cathode, can guide the beam through the achromatic dogleg into the ELBE Linac. Alternatively, the electron beam reaches the $180^{\circ}$-bending dipole for energy and energy spread measurements. Beam position monitors and steerers are not shown here.

UV laser and the diagnostic beam line [22] (schematically shown in Fig. 2). The whole diagnostic beam line is five meters long. At the exit of the gun is the solenoid, followed by the laser input port and a Faraday cup. The quadrupole triplet can be used to optimize the beam before the $45^{\circ}$ bending dipole. Several beam position monitors and alignment coils (steerers) can guide the beam. Seven screens can be inserted along the beam line to diagnose the beam spot. A $180^{\circ}$ dipole ( $\mathrm{C}$ bend) is installed at the end of the first straight line for energy and energy spread measurement.

The rf source of the gun is a solid-state amplifier, which can work in cw mode or pulsed mode. For some measurements, the macropulsed mode was used in order to operate the gun at higher gradient than in cw mode, while maintaining low liquid helium consumption. The macropulse length was typically between 10 and $20 \mathrm{~ms}$ and the repetition rate was $1 \mathrm{~Hz}$.

\section{B. Experiments and measurement results}

By focusing the dark current electrons on screen 1 $(1.5 \mathrm{~m})$ or screen $2(3 \mathrm{~m})$ with the solenoid $(1 \mathrm{~m})$, one can image the cathode and the cathode hole. The dark current image is an important tool to study and understand the field emission sources in rf guns [14,16]. Figure 3 is a picture of the dark current from the gun with $\mathrm{Cs}_{2} \mathrm{Te}$ photocathode. The round circle is believed to show the field emission's initial source on the cathode edge and the border of the cavity's cathode hole, and besides the clear circle there are still some "halo clouds," which changes the profiles and brightness versus the solenoid current. They are possibly from the electrons with lower energy. A bright spot inside of the circle is formed mainly from the $\mathrm{Cs}_{2} \mathrm{Te}$ coating and the cathode-plug surface.

The dark current intensity was measured using the Faraday cup which is located at a distance of $1.4 \mathrm{~m}$ from the cathode. For the pulsed mode, the current in the pulse 


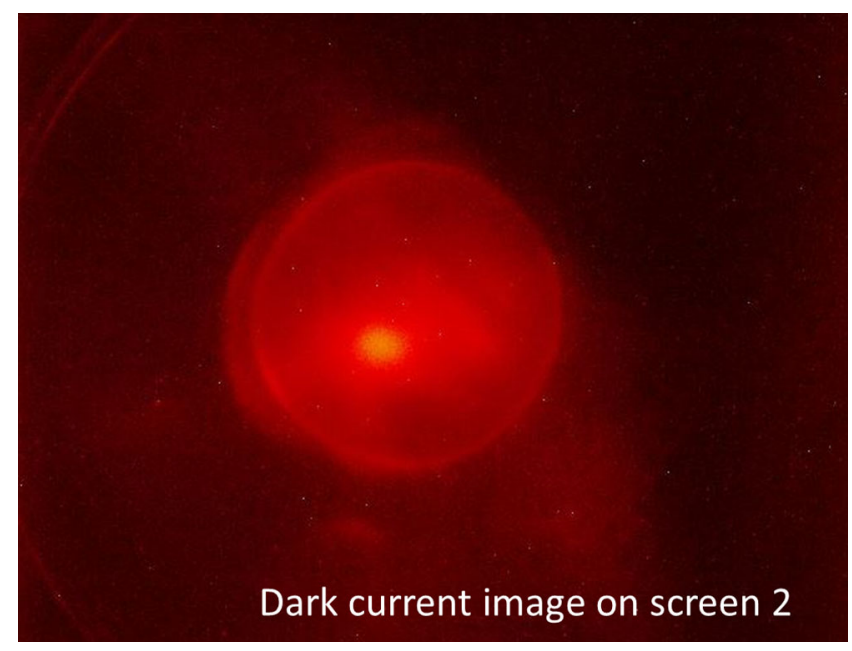

FIG. 3. The image of the dark current focused with the solenoid on the YAG screen 2.

was measured by a $10 \mathrm{k} \Omega$ resistor with an oscilloscope. In Fig. 4 the Faraday cup signal on the oscilloscope, the trigger of the cathode drive laser and the macropulse signal are displayed. The dark current shows a plateau, delivering the signal with the same time structure as that of the cavity gradient. The narrow peaks are believed to originate from multipacting at low gradient. The photoelectrons form a peak on the plateau, depending on the temporal structure and the pulse energy of the driven laser. Actually, when the SRF gun is routinely operated, the photocurrent peak is several magnitudes higher than the dark current peak. In order to take this picture, the power of the cathode drive laser was reduced significantly so that the photocurrent and

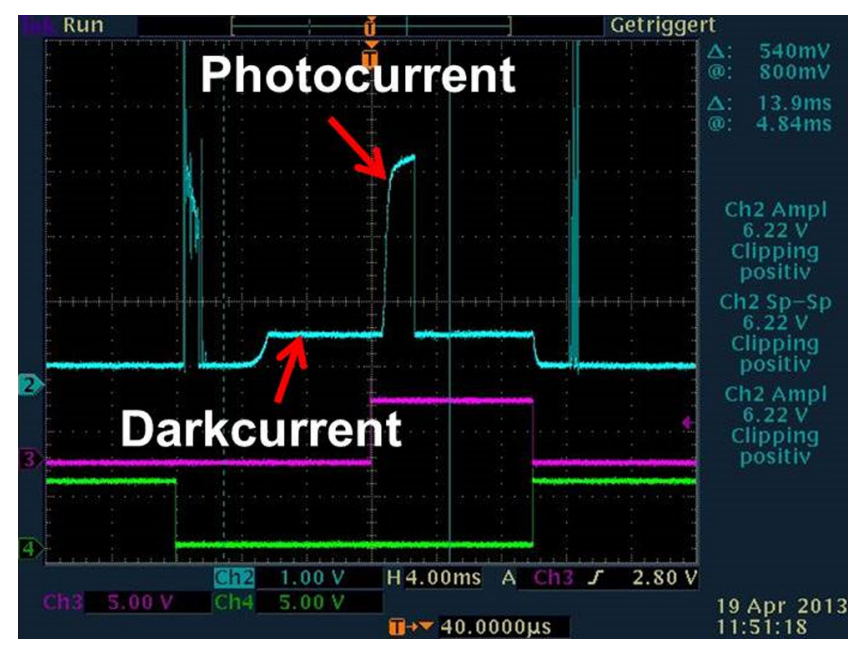

FIG. 4. Display on oscilloscope, $E_{z \cdot \max }=16.2 \mathrm{MV} / \mathrm{m}$. The blue line (top) gives out the Faraday cup signal of the sum of the dark current and the photocurrent; the green line (bottom) shows the macropulse signal of the solid state amplifier; and the magenta line (middle) represents the trigger signal of the cathode drive laser. the dark current could be shown clearly on the oscilloscope screen.

One of the aims of the experiment is to distinguish the dark current emitted from the cavity and that from the photocathode. The experiment was done in the following procedure. At first the current, energy and energy spectrum of the dark current from the gun without any cathode plug was logged down. Then, a photocathode was inserted into the cavity through the cathode hole on the back side and the cavity was retuned to $1300 \mathrm{MHz}$. The measurements were repeated for different cathode materials. The photocathode transfer system allows us to exchange cathodes quickly without warming up the cavity. Therefore the measurement for different photocathode materials could be done in a short time cycle.

A niobium cathode coated with lead, a pure molybdenum cathode and two cathodes with $\mathrm{Cs}_{2} \mathrm{Te}$ layer on Mo plugs were measured. Just as the typical field emission, the dark current increased with rising rf field. In Fig. 5 the results of all measurements are collected. They all show a similar exponential trend. It is readily clear that the largest part of the dark current came from the cavity itself, because the difference between the dark current of the gun with a $\mathrm{Cs}_{2}$ Te photocathode and that without cathode is less than $30 \%$ of the total value. However, the metal cathodes do not provide obvious dark current. The results will be discussed by using the field emission theory in Sec. III C.

The results of the energy distribution give out more information about the dark current sources. In principle the energy of the electron beam can be measured by using the

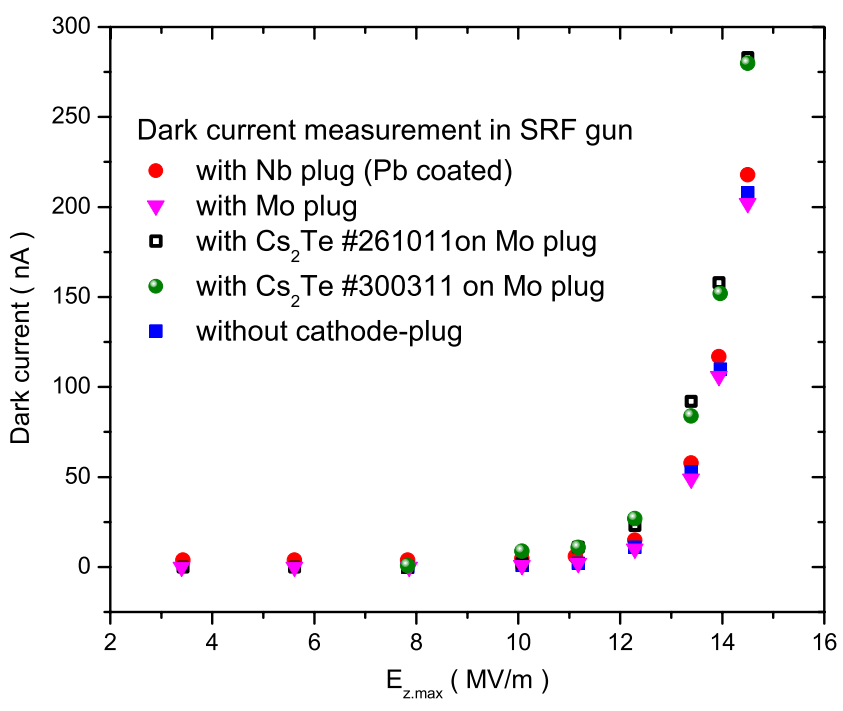

FIG. 5. Dark current from the gun without any cathode and with different photocathodes vs axis peak field. The red dots are from a measurement with a $\mathrm{Pb}$-coated $\mathrm{Nb}$ cathode. Pink triangles represent the case with uncoated Mo cathode. The hollow squares and the green balls are with $\mathrm{Cs}_{2} \mathrm{Te}$ emission layer on Mo substratum. The blue squares give the result of the case without cathode plug. 
$180^{\circ}$-bending dipole at $5 \mathrm{~m}$ downstream from the cathode surface. But the low energy part of the dark current could not be efficiently transported to $5 \mathrm{~m}$ forward. Thus, the $45^{\circ}$-bending dipole ( $2 \mathrm{~m}$ away from the gun exit) was used to measure the energy spread of the dark current. Figure 6 shows the energy spectra with intensity normalized to the total dark current measured with the Faraday cup. All curves show similar multipeak structures. As known in the sinusoidal rf field, the field induced emission happens around the $90^{\circ} \mathrm{rf}$ phase. The electrons emitted near the cathode are at the right accelerating of phase and therefore gain the maximum energy, just like the photocurrent beam at the proper rf phase. Simulation with ASTRA track code [23] shows that a part of the electrons emitted too late in the rf phase could be trapped in one of the cells. Those that are not eliminated by collisions with the cavity wall can still be accelerated during the next rf cycle and generate the other peaks at lower energy. Also the electrons emitting from the cavity iris can experience only a part of acceleration field and attain lower energy. One has to pay attention to the highest energy peak, because its energy is very close to that of photoelectrons, making it difficult to separate these two beams.

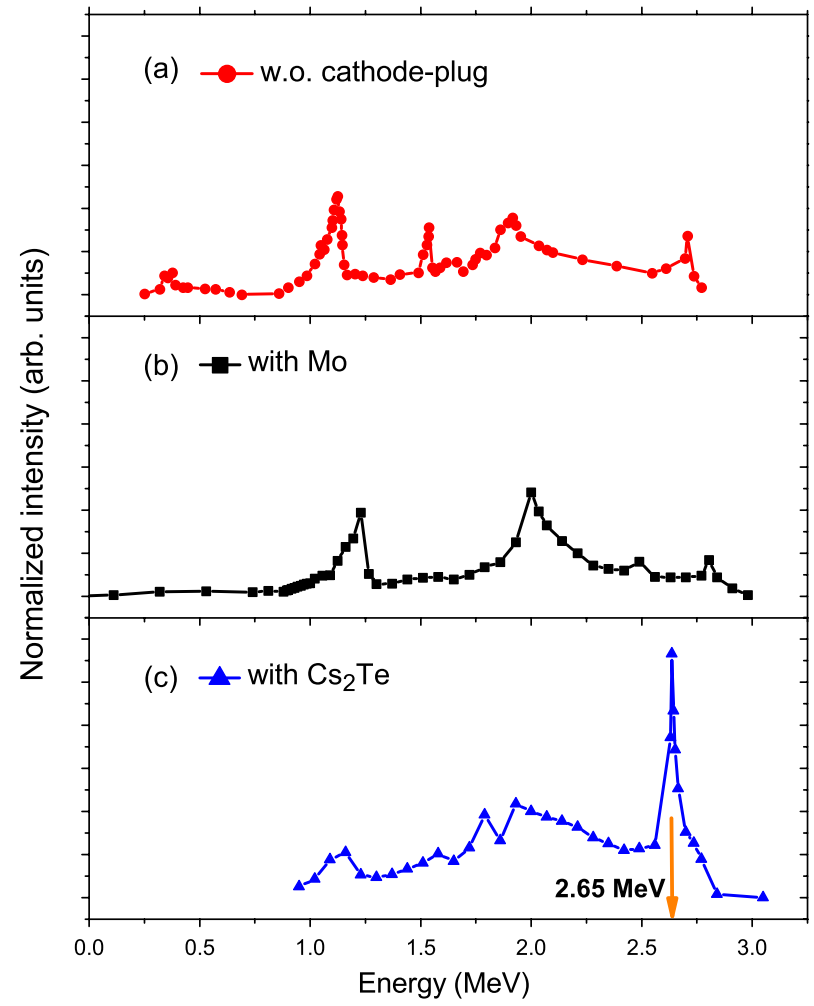

FIG. 6. Energy spectra of the dark current from the SRF gun with $E_{z . \max }=16.2 \mathrm{MV} / \mathrm{m}$. The intensity is normalized to the total dark current. The curve in (a) represents the case only from the $\mathrm{Nb}$ cavity. Part (b) shows the result of the cavity with Mo plug, and part (c) is from the case of the cavity with $\mathrm{Cs}_{2} \mathrm{Te}$ photocathode. The orange arrow points out the measured photoelectron energy.
The main peaks of the spectra in Figs. 6(a) and 6(b) are similar. As shown in Fig. 5, there is no field emission from the Mo cathode. Besides the lower energy peaks, diagram (c) shows a new emission, which must be caused by the $\mathrm{Cs}_{2}$ Te layer. This peak shows the energy of $2.65 \mathrm{MeV}$, which is the same as that of the photo beam. The integrated intensity of this peak is $16.3 \%$ of the total area. The dark current from the cathode has such a narrow energy distribution, because the field emitted electrons gain the initial kinetic energy due to the cathode bias voltage to be accelerated efficiently like the photo beam.

In order to understand the effect of the cathode bias voltage and rf field on the dark current, we studied the cavity with Mo plug and that with $\mathrm{Cs}_{2} \mathrm{Te}$ cathode in detail. The dark current from the gun with Mo cathode has been measured again at higher gradient (Fig. 7), which represents the same exponential trend as in Fig. 5. The experimental data is used for the analysis in the following section. For a certain cavity gradient, the cathode biasvoltage is varied to verify its effect on the dark current. However, from Fig. 8 the dark current intensity stays constant with varying cathode bias voltage.

But if one checks the energy distribution of the dark current, the effect of various cathode voltages can be observed. In Fig. 9, with increasing rf field, the spectra look relatively similar with higher intensity and slightly shifted peak positions. For the test at a gradient of 16.2 MV/m shown in the middle diagram, one can see the difference due to the cathode voltages. When the cathode voltage rises from 3 to $6 \mathrm{kV}$, the peak at $2.8 \mathrm{MeV}$ increases and the peak at $1.2 \mathrm{MeV}$ decreases. In the currently accessible gradient and bias range, a higher cathode voltage does not generate new emission but changes the energy distribution of the dark current.

Usually the SRF gun is operated with a $\mathrm{Cs}_{2} \mathrm{Te}$ photocathode [see the Fig. 6(c)]. We measured the spectra under

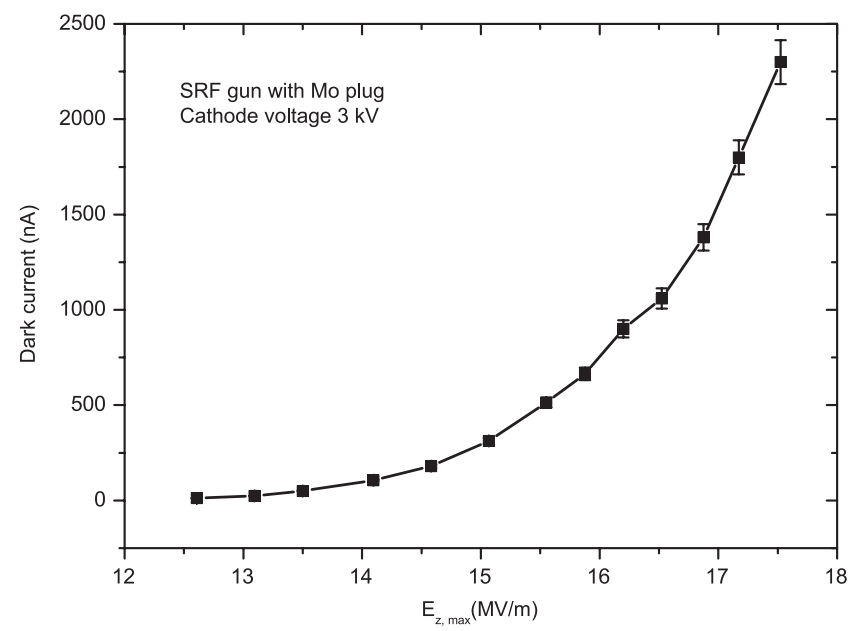

FIG. 7. Dark current vs peak field $E_{z \text { max }}$ for the cavity with Mo cathode. Measurement was done with pulsed mode. 


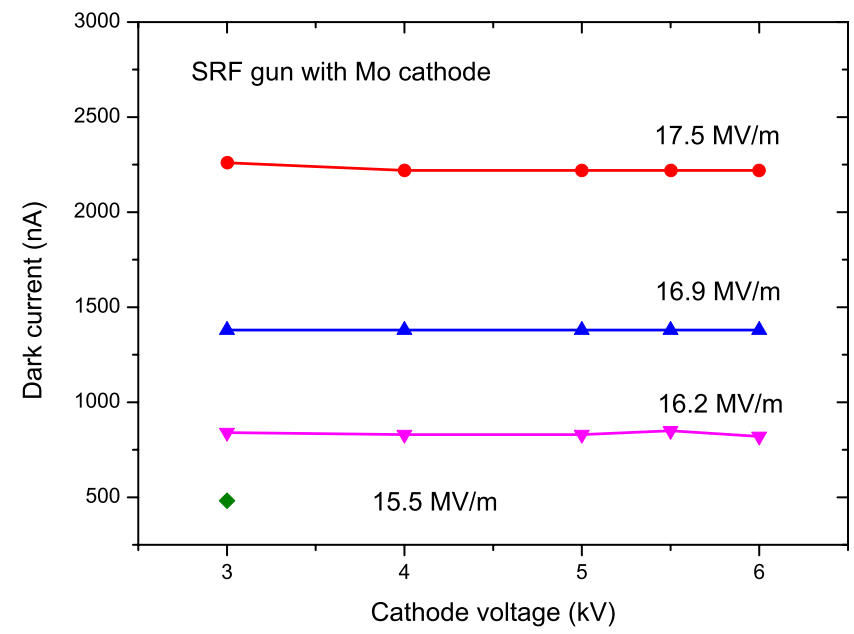

FIG. 8. Dark current vs cathode voltage for the cavity with Mo cathode.

various gradients (Fig. 10) to check the distribution dependence. The intensity has been normalized with respect to the dark current measured by the Faraday cup. It is readily found that the spectra are shifted to the high energy direction according to the increasing field. As known from Fig. 6, the lower energy peaks are formed by the emission from the cavity itself and most of the

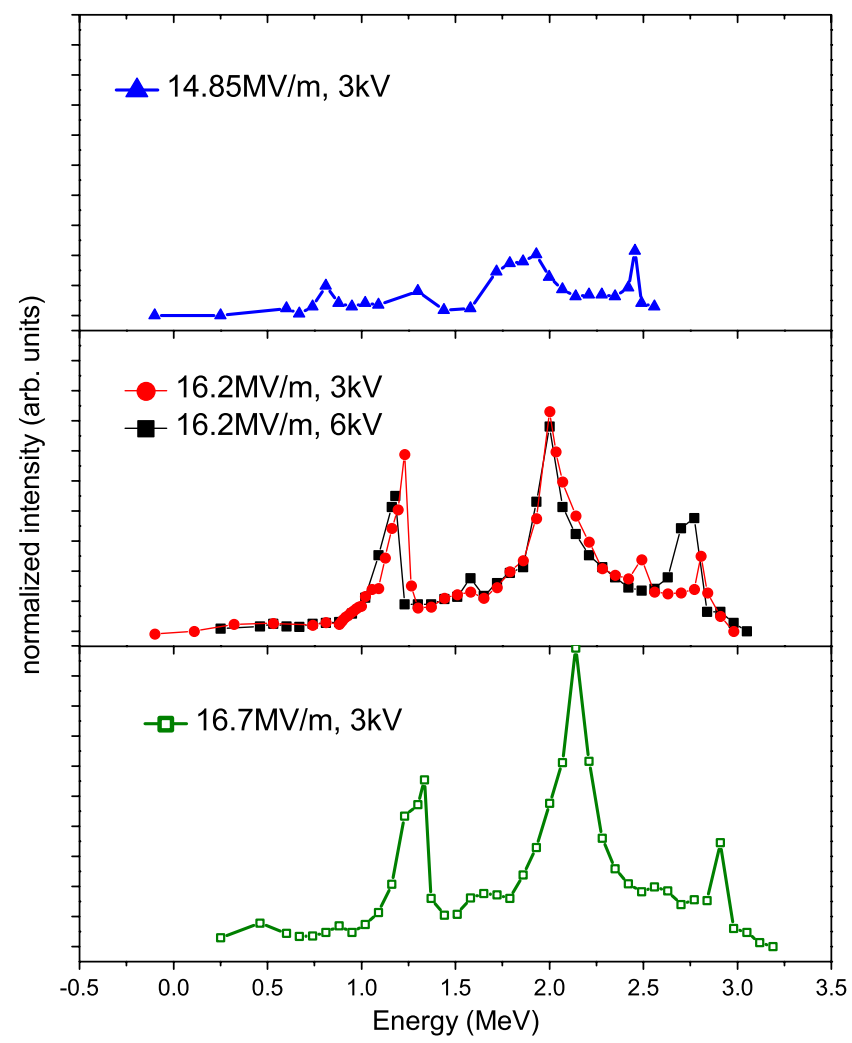

FIG. 9. Energy spectra of the dark current from the SRF gun with Mo cathode plug. The measurement was done with various rf fields $E_{z \cdot \max }$ and cathode voltages.

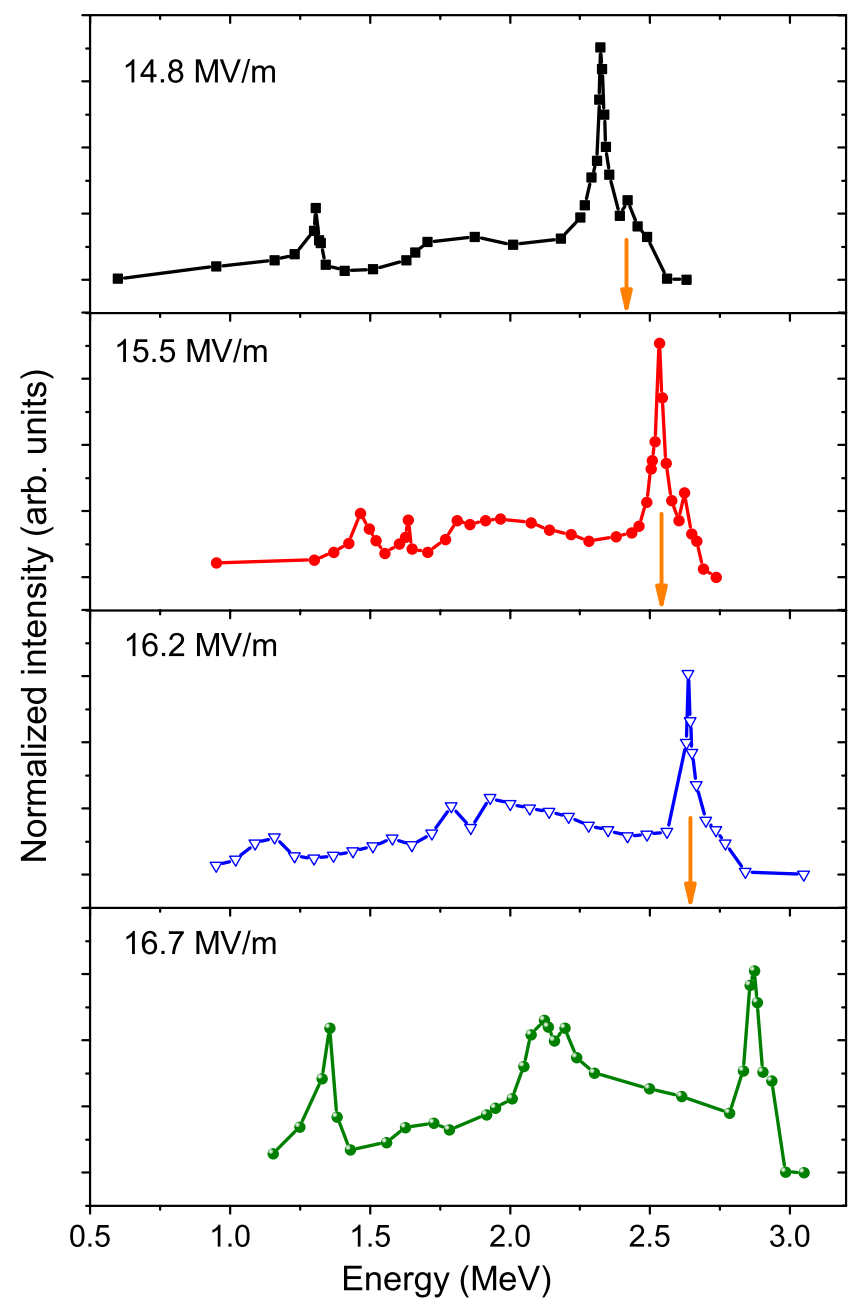

FIG. 10. Energy spectra of the dark current vs gradient for the SRF gun with the same $\mathrm{Cs}_{2}$ Te photocathode. The cathode voltage was set to $3 \mathrm{kV}$. The orange arrows indicate the energy of the photoelectron beam (laser phase $30^{\circ}$ ).

highest energy peak comes from the $\mathrm{Cs}_{2} \mathrm{Te}$ photocathode. Although the intensity of total dark current rises quickly with the increasing gradient, the peak at highest energy seems to be constant. Actually, one can calculate $\eta=$ $N_{\text {peak }} / N_{\text {total }}$, where $N_{\text {peak }}$ is the particle numbers inside the highest energy peak with an energy spread of $\Delta E=140 \mathrm{keV}$, and $N_{\text {total }}$ is the number of the total particles (listed in Table I). The result shows that the dark current with highest energy increased more slowly than the total one.

TABLE I. The statistics of the energy spectrum in Fig. 10.

\begin{tabular}{lcc}
\hline \hline$E_{\text {z. max }}(\mathrm{MV} / \mathrm{m})$ & Peak $@(\mathrm{MeV})$ & $\eta=N_{\text {peak }} / N_{\text {total }}(\%)$ \\
\hline 14.8 & 2.3 & 24.6 \\
15.5 & 2.53 & 20.8 \\
16.2 & 2.64 & 16.3 \\
16.7 & 2.87 & 12.9 \\
\hline \hline
\end{tabular}


As described in Fig. 2, the photo beam is bent by the dipole through the dogleg beam line and then enters the linac afterwards. At the same time, only a small part of the field emitted electrons in a narrow energy window can reach the linac while the others with lower energy will be lost in the beam line.

However, the next generation SRF gun is operated with a new cavity which may have gradients more than double the existing one. The dark current emitted from the new cavity and that from the $\mathrm{Cs}_{2} \mathrm{Te}$ photocathode are therefore going to be different, which will be analyzed later.

\section{Analysis}

Basically the dark current is generated by the field emission, which can be analyzed via the well-known Fowler-Nordheim (F-N) theory. The field emission current in an rf field $E=E_{0} \sin \theta$ (where $\theta$ is rf phase) has been analyzed based on the F-N equation in Ref. [24]

$$
\begin{aligned}
I= & \frac{5.7 \times 10^{-12} \times 10^{4.52 \Phi^{-0.5}} A_{0}\left(\beta E_{0}\right)^{2.5}}{\Phi^{1.75}} \\
& \times \exp \left(-\frac{6.53 \times 10^{9} \Phi^{1.5}}{\beta E_{0}}\right),
\end{aligned}
$$

where $I$ is the time-averaged dark current (in A), $\Phi$ is the work function of the emitting material (in eV), $E_{0}$ is the amplitude of the sinusoidal macroscopic electric surface field (in $\mathrm{V} / \mathrm{m}$ ), $A_{0}$ is the emission area (in $\mathrm{m}^{2}$ ), and $\beta$ is the field enhancement factor. $\beta E_{0}$ introduces the local field variation due to the microscopic surface roughness via scaling factor $\beta$. According to the former studies [25], in a superconducting cavity, typical $\beta$ is several hundred.

The natural logarithm of $I / E_{0}^{2.5}$ gives out

$$
\begin{aligned}
\ln \left(\frac{I}{E_{0} 2.5}\right)= & \ln \left(\frac{5.7 \times 10^{-12} \times 10^{4.52 \Phi^{-0.5}} A_{0} \beta^{2.5}}{\Phi^{1.75}}\right) \\
& -\frac{6.53 \times 10^{9} \Phi^{1.5}}{\beta E_{0}} .
\end{aligned}
$$

The graphical presentation of the measurement data in the way of Eq. (2) is the F-N plot. A linear fit can be used to calculate the enhancement factor $\beta$ from the slope, and the size $A_{0}$ from the intercept:

$$
\frac{d\left(\log _{10}\left(I / E_{0}^{2.5}\right)\right)}{d\left(1 / E_{0}\right)}=-\frac{2.84 \times 10^{9} \Phi^{1.5}}{\beta} .
$$

From the current measurement presented in Fig. 7, one can draw the F-N plot and perform a linear fit (Fig. 11). As the work function $\Phi$ of pure niobium is $4.3 \mathrm{eV}$ [26], the field enhancement factor can be calculated as $\beta=239$, and the effective emission area $A_{0}=1.6 \times 10^{-15} \mathrm{~m}^{2}$. This

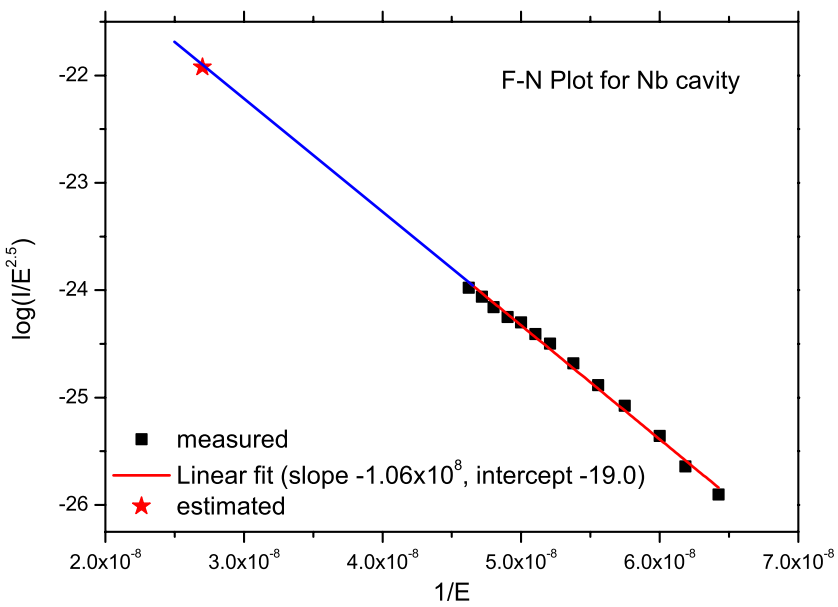

FIG. 11. F-N plot of the dark current from the niobium cavity. In the horizontal axis $E(\mathrm{~V} / \mathrm{m})$ is the $E$-field amplitude. The star is the data extended from the linear fit with respect to $E_{z \cdot \max }=30 \mathrm{MV} / \mathrm{m}, I=1 \times 10^{-3} \mathrm{~A}$.

result represents the "global" trace of the field emission in the superconducting cavity, the integrated effect of several jagged whiskers or sharp projections. Based on this calculation, one can estimate the dark current from the same cavity with higher gradient (extended from the linear fitting in Fig. 11).

However, one must keep in mind that the detected dark current is only the part through the gun exit and measured by the Faraday cup. The real field emission inside the cavity can be higher.

On the other hand, the situation for the $\mathrm{Cs}_{2} \mathrm{Te}$ cathode is more complicated. From Figs. 5 and 10, the dark current from $\mathrm{Cs}_{2} \mathrm{Te}$ increases with the rising gradient, but this increase is much less than the change of the dark current from cavity itself. There is no published work to study the field emission phenomena of $\mathrm{Cs}_{2} \mathrm{Te}$ film. Although there are a lot of reports about the dark current of rf guns, the emission from cavity and that from cathode were mostly combined.

The classic F-N theory based on the free electron model cannot be applied for semiconductor cathodes. In Refs. $[27,28]$ the F-N theory was restated to describe the field emission on the semiconductors. Field-induced emission through pure dielectric films does not always follow the F-N equation like in the case of metal, but falls into several regimes: (1) F-N form at low field range, (2) smooth transition regime, (3) "saturation" at high field zone and (4) explosion after one threshold. However, the field at which the switch occurs between two regimes depends on the film thickness, the work function, and the surface state of the semiconductor.

As shown in Fig. 5, the difference between the curve with $\mathrm{Cs}_{2} \mathrm{Te}$ cathode and that with Mo-plug delivers dark current from the $\mathrm{Cs}_{2} \mathrm{Te}$ layer. The relationship of this dark current versus field follows the F-N form. Compared with 
the normal conducting rf gun like in PITZ [13], our experiments in SRF gun use relative low field on cathode, and we assume that our experiment is still in the first regime of "F-N form." For the field emitter on the $\mathrm{Cs}_{2} \mathrm{Te}$ layer, a work function of $4.75 \mathrm{eV}$ can be applied, the field enhancement factor is 639 , and the emission area is $3.5 \times 10^{-19} \mathrm{~m}^{2}$. However, it is difficult to predict the dark current from $\mathrm{Cs}_{2} \mathrm{Te}$ at higher gradient. From the practical experience of the DESY PITZ gun, the dark current directly from the $\mathrm{Cs}_{2} \mathrm{Te}$ film was reported to $10 \mu \mathrm{A}$ at about $40 \mathrm{MV} / \mathrm{m}$ on the cathode [13].

From the former analysis the conclusion can be attained that the field emission of the cavity is the main source for the dark current of the ELBE SRF gun.

\section{KICKER OF THE DARK CURRENT}

The dark current is an undesired and inevitable phenomenon in $\mathrm{rf}$ guns and SRF guns, especially relevant when operating at high acceleration gradients. In order to reduce the dark current before it enters the accelerators, a kicker in front of the linac beam line can be applied. For example, at FLASH a dark current kicker has been employed and reduces significantly the dark current intensity downstream [18].

For the kicker for SRF gun at ELBE, it should operate in $13 \mathrm{MHz} \mathrm{cw}$ mode and with ns pulse duration. The SLACtype kicker [29] is considered to be installed between the
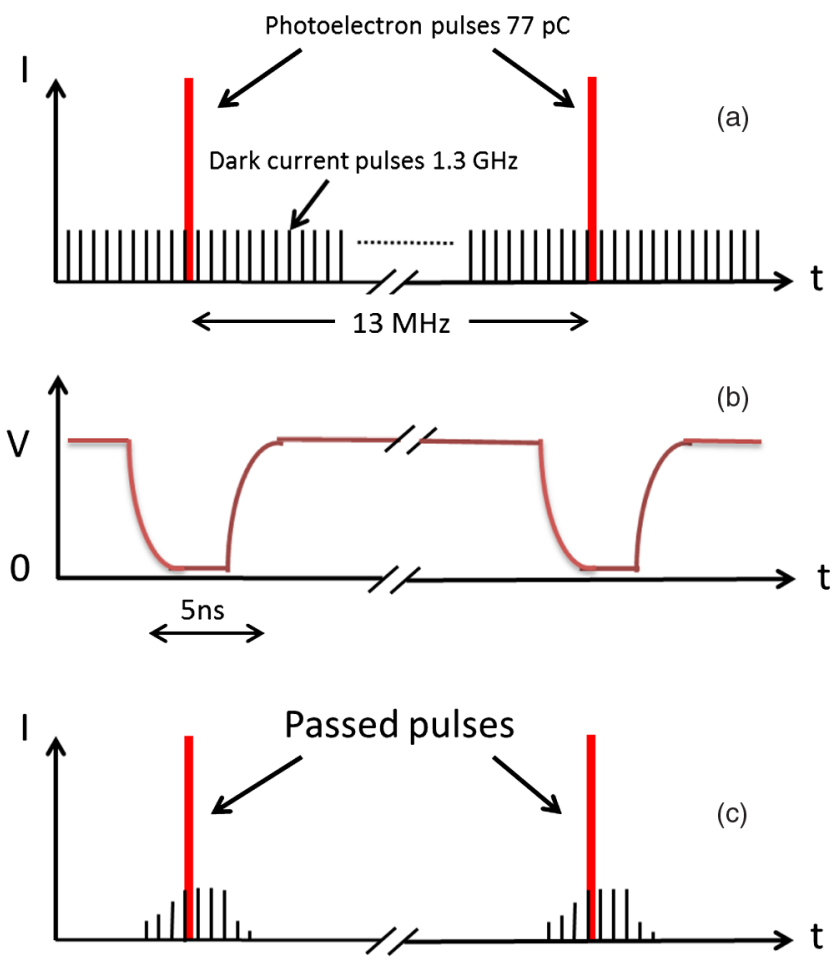

FIG. 12. The basic concept of the kicker designed for the SRF gun [19].

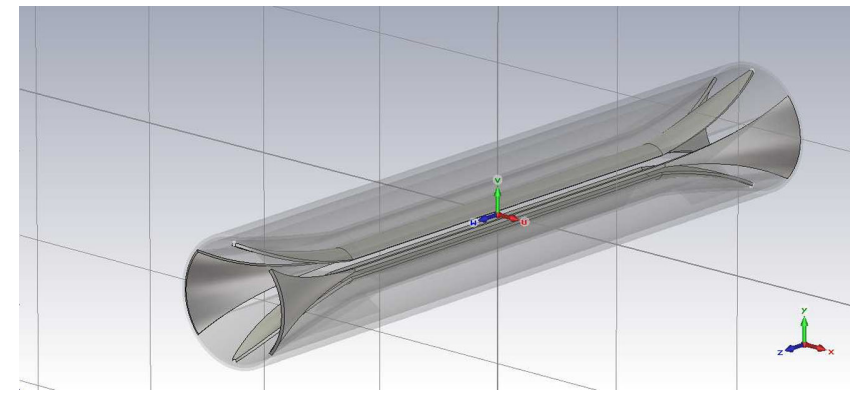

FIG. 13. The model of the strip line kicker for the SRF gun, which is $250 \mathrm{~mm}$ long with a $150 \mathrm{~mm}$ strip and $50 \mathrm{~mm}$ taper at each side. The structure consists of two strip lines with four $50 \Omega$ ports and two ground fenders for homogenizing the field inside the chamber.

gun and the dogleg beam line. The proposed concept is shown in Fig. 12. The $13 \mathrm{MHz}$ photoelectron pulses together with the $1.3 \mathrm{GHz}$ dark current bunches enter into the beam line (a). The fast kicker (strip line Kicker) is applied with long pulses of $13 \mathrm{MHz}$ and $5 \mathrm{~ns}$ windows (b) resulting in $93.5 \%$ of the dark current being kicked out. The passed beam consists of $13 \mathrm{MHz}$ photoelectron pulses together with dark current pulses inside $5 \mathrm{~ns}$ (c).

The structure of the kicker is shown in Fig. 13. The total model is $250 \mathrm{~mm}$ long with $150 \mathrm{~mm}$ strip line, and a radius of the kicker chamber of $5 \mathrm{~mm}$. A driver with $\pm 500 \mathrm{~V}$ pulses will be applied on the ports. The CST simulation has been done to look into the paths of the particles through the kicker. Electrons with energy of $5 \mathrm{MeV}$ will be deflected by $1.06^{\circ}$ away from the axis to the collimator downstream.

\section{CONCLUSION AND OUTLOOK}

In summary, the experimental investigations and analysis of the dark current from the SRF gun have been reported. The dark current rises exponentially with the cavity gradient. It has a multipeaked energy spectrum and the highest energy is compatible to that of photocurrent. The contribution of the niobium cavity and the photocathode material has been studied. The field emission from the $\mathrm{Nb}$ cavity, the main source of the dark current in SRF gun, has been analyzed based on the F-N theory. At last, a proposal of a strip line kicker to deflect most of the dark current has been discussed.

In cooperation with JLab two new $3+1 / 2$ cell cavities with the same geometric structure but an improved quality have been produced. In the next step, a new upgraded SRF gun will replace the existing SRF gun, which will significantly improve the photo beam quality. However, one has to pay attention to the possible increased dark current from the new gun as well. The dark current study for the new SRF gun will be continued. 


\section{ACKNOWLEDGMENTS}

We would like to thank the whole ELBE team for their help and assistance with this work. We acknowledge the support of the European Community-Research Infrastructure Activity under the FP7 program (EuCARD, Contract No. 227579), as well as the support of the German Federal Ministry of Education and Research Grant No. 05 ES4BR1/8.

[1] F. Stephan, C. H. Boulware et al., Phys. Rev. ST Accel. Beams 13, 020704 (2010).

[2] I. Bazarov, A. Kim, M. Lakshmanan, and J. Maxson, Phys. Rev. ST Accel. Beams 14, 072001 (2011).

[3] D. J. Gibson, F. Albert, S. G. Anderson, S. M. Betts, M. J. Messerly, H. H. Phan, V. A. Semenov, M. Y. Shverdin, A. M. Tremaine, F. V. Hartemann, C. W. Siders, D. P. McNabb, and C. P. J. Barty, Phys. Rev. ST Accel. Beams 13, 070703 (2010).

[4] C. Hernandez-Garcia and T. Kamps, at the Workshop of Future Light Sources 2012, Newport News, Virginia, USA.

[5] A. Arnold and J. Teichert, Phys. Rev. ST Accel. Beams 14, 024801 (2011).

[6] R. Xiang, A. Arnold, H. Buettig, D. Janssen, M. Justus, U. Lehnert, P. Michel, P. Murcek, A. Schamlott, Ch. Schneider, R. Schurig, F. Staufenbiel, and J. Teichert, in Proceedings of the XIIIth International Workshop on Polarized Sources, Targets \& Polarimetry (World Scientific, Singapore, 2011), pp. 249-256.

[7] A. Arnold, H. Buettig, D. Janssen, T. Kamps, G. Klemz, W. D. Lehmann, U. Lehnert, D. Lipka, F. Marhauser, P. Michel, K. Moeller, P. Murcek, Ch. Schneider, R. Schurig, F. Staufenbiel, J. Stephan, J. Teichert, V. Volkov, I. Will, and R. Xiang, Nucl. Instrum. Methods Phys. Res., Sect. A 577, 440 (2007).

[8] A. Arnold, H. Buettig, D. Janssen, T. Kamps, G. Klemz, W. D. Lehmann, U. Lehnert, D. Lipka, F. Marhauser, P. Michel, K. Moeller, P. Murcek, Ch. Schneider, R. Schurig, F. Staufenbiel, J. Stephan, J. Teichert, V. Volkov, I. Will, and R. Xiang, Nucl. Instrum. Methods Phys. Res., Sect. A 593, 57 (2008).

[9] J. Teichert, A. Arnold, H. Büttig, M. Justus, T. Kamps, U. Lehnert, P. Lu, P. Michel, P. Murcek, J. Rudolph, R. Schurig, W. Seidel, H. Vennekate, I. Will, and R. Xiang, Nucl. Instrum. Methods Phys. Res., Sect. A 743, 114 (2014).

[10] R. Xiang, A. Arnold, H. Buettig, D. Janssen, M. Justus, U. Lehnert, P. Michel, P. Murcek, A. Schamlott, Ch. Schneider, R. Schurig, F. Staufenbiel, and J. Teichert, Phys. Rev. ST Accel. Beams 13, 043501 (2010).

[11] J. H. Han, J. Baehr, H.-J. Grabosch, M. Krasilnikov, V. Miltchev, A. Oppelt, B. Petrosyan, S. Riemann, L. Staykov, F. Stephan, M. V. Hartrott, K. Floettmann, S. Schreiber, J. Roensch, P. Michelato, L. Monaco, and D. Sertore, in
Proceedings of the 21st Particle Accelerator Conference, Knoxville, 2005 (IEEE, Piscataway, NJ, 2005), pp. 895-897.

[12] I. Bohnet, J. H. Han, M. Krasilnikov, F. Stephan, and K. Floettmann, in Proceedings of Sixth European Workshop on Beam Diagnostics and Instrumentation for Particle Accelerators, Mainz, Germany (JACoW, GSI, Mainz, 2003), pp. 242-244.

[13] I. Isaev, P. Boonpornprasert et al., in Proceedings of the 35th International Free Electron Laser Conference, New York, USA (JACoW, New York, 2013), pp. 282-286.

[14] D. H. Dowell, E. Jongewaard, C. Limborg-Deprey, J. F. Schmerge, and A. Vlieks, in Proceedings of the 22nd Particle Accelerator Conference, Albuquerque, New Mexico (IEEE, New York, 2007), pp. 1299-1301.

[15] L. Foehlich, in Proceedings of the 22nd Particle Accelerator Conference, Albuquerque, New Mexico (Ref. [14]), pp. 956-958.

[16] L. Monaco, P. Michelato, C. Pagani, P. Pierini, D. Sertore, J. H. Han, S. Schreiber, M. Krasilnikov, and F. Stephan, in Proceedings of the 10th European Particle Accelerator Conference, Edinburgh, Scotland, 2006 (EPS-AG, Edinburgh, Scotland, 2006), pp. 2493-2495.

[17] J. H. Han, M. Krasilnikov, and K. Flöttmann, Phys. Rev. ST Accel. Beams 8, 033501 (2005).

[18] D. Lipka, W. Kleen, J. Lund-Nielsen, D. Nölle, S. Vilcins, and V. Vogel, in Proceedings of the 10th European Workshop on Beam Diagnostics and Instrumentation for Particle Accelerators, Hamburg, Germany (DESY, Hamburg, 2011), pp. 572-574.

[19] J. Teichert, A. Arnold, P. Murcek, G. Staats, R. Xiang, P. Lu, H. Vennekate, R. Barday, and T. Kamps, in Proceedings of the 53th ICFA Advanced Beam Dynamics Workshop on Energy Recovery Linacs, Novosibirsk, Russia (JACoW, Novosibirsk, 2013), pp. 75-79.

[20] R. Xiang, A. Arnold, P. Lu, P. Murcek, J. Teichert, H. Vennekate, R. Barday, T. Kamps, V. Volkov, in Proceedings of the 35th International Free Electron Laser Conference, New York, USA (JACoW, New York, 2013), pp. 455-457.

[21] Los Alamos Accelerator Code Group, http://laacg.lanl.gov/ laacg/services/services.phtml.

[22] T. Kamps, A. Arnold, D. Boehlick, M. Dirsat, G. Klemz, D. Lipka, T. Quast, J. Rudolph, M. Schenk, F. Staufenbiel, J. Teichert, and I. Will, Rev. Sci. Instrum. 79, 093301 (2008).

[23] K. Floettmann, http://www.desy.de/ mpyflo/.

[24] J. W. Wang and G. A. Loew, Report No. SLAC-PUB-7684, 1997.

[25] H. Pamamsee, J. Knobloch, and T. Hays, RF Superconductivity for Accelerators (Wiley, New York, 1998).

[26] H. B. Michaelson, J. Appl. Phys. 48, 4729 (1977).

[27] R. G. Forbes, Ultramicroscopy 79, 11 (1999).

[28] R. G. Forbes, Solid State Electron. 45, 779 (2001).

[29] A. Krasnykh, at the ILC Damping Ring R\&D Workshop, Ithaca, NY, USA (Report No. SLAC-WP-077, 2006). 